\title{
Fijación de fractura de columna posterior de acetábulo, asistida por artroscopía
}

\author{
Fixation of acetabulum posterior column fracture, \\ arthroscopy assisted
}

\author{
Altamirano-Cruz MA, ,*, Flores-Villalobos A,*,\$ \\ Rodríguez-Lara SQ," Bustos-Mora R, ${ }^{* \|}$ Gutiérrez-Ramírez LX*,**
}

Unidad Médica de Alta Especialidad, Centro Médico Nacional de Occidente, Instituto Mexicano del Seguro Social.

RESUMEN. La cirugía artroscópica de la cadera ha evolucionado considerablemente en los últimos años, ofreciendo oportunidad de tratamiento a muchas patologías intraarticulares no reconocidas previamente. Con avances recientes en métodos e instrumentos quirúrgicos, se realiza cada vez más frecuentemente, con indicaciones que incluyen: diagnóstico de pacientes con dolor de cadera, lesiones del labrum acetabular, extracción de cuerpos extraños, desbridamiento en artritis séptica, pinzamiento femoroacetabular y algunos daños extraarticulares. Otra patología intraarticular de la cadera que se beneficia de la asistencia por artroscopía se podría encontrar en el manejo de fracturas acetabulares simples. Reportamos el caso de un paciente con fractura de columna posterior acetabular, tratado vía artroscópica, y los resultados obtenidos. Las técnicas artroscópicas para la fijación de fracturas intraarticulares ofrecen la ventaja de una visualización y reducción superiores de la superficie articular, ya que se hacen mediante observación del foco de fractura, permitiendo una reducción anatómica. Además, las técnicas de reducción artroscópica ofrecen otro beneficio para las lesiones intraarticulares, como en este caso la lesión de labrum posterior. En los trazos simples con poco desplazamiento de las fracturas de columna posterior
ABSTRACT. Arthroscopic surgery of the hip has evolved significantly over the last years, offering an opportunity of treatment in several intra-articular pathologies. Recent methods and instrumental advances have allowed more frequent use of this procedure, with various indications such as: hip pain, acetabular labrum lesions, extraction of foreign bodies, debridement in septic arthritis, pincer impingement, and some extraarticular injuries. Another intra-articular hip pathology that benefits from arthroscopic assistance could be found in the management of simple acetabular fractures. Therefore, this report presents the case of a patient with a posterior column fracture treated with an arthroscopic approach. Arthroscopic techniques for the fixation of intra-articular fractures offer the advantage of a direct visualization of the articular surface, which results in an anatomical reduction. In addition, arthroscopic techniques also present a benefit for intra-articular injuries such as the posterior labral lesion in this case. In simple fractures of the posterior acetabular column with minimum displacement, where percutaneous screw fixation is useful as a definitive fixation method, hip arthroscopy is useful for the reduction process and verifies the extra-articular screw trajectory.

\footnotetext{
* Unidad Médica de Alta Especialidad, Centro Médico Nacional de Occidente, Instituto Mexicano del Seguro Social.

‡ Médico adscrito al departamento de traumatología y ortopedia. Módulo de politrauma, pelvis y acetábulo. Guadalajara, Jalisco, México.

${ }^{\S}$ Médico residente de tercer año de traumatología y ortopedia. Guadalajara, Jalisco, México.

" Universidad Autónoma de Guadalajara. Doctor en farmacología. Investigador. Guadalajara, Jalisco, México.

" Médico adscrito al departamento de politrauma y cirugía articular. Guadalajara, Jalisco, México.

** Jefe de servicio de traumatología y ortopedia. Guadalajara, Jalisco, México.
}

Dirección para correspondencia:

Dr. Marco Antonio Altamirano-Cruz

Av. José María Morelos Núm. 2060, Col. Ladrón de Guevara, CP 45600, Guadalajara, Jalisco, México.

E-mail: marco.trauma@gmail.com 
acetabular, donde la fijación percutánea con tornillos es útil como método de fijación definitiva, la artroscopía de cadera es de utilidad con el fin de asistir durante el proceso de reducción y verificar que el trayecto del tornillo no se encuentre intraarticular.

Palabras clave: Artroscopía de cadera, fractura, acetábulo, mínima invasión.

\section{Introducción}

La cirugía artroscópica de cadera es un procedimiento técnicamente demandante, que requiere la aplicación de tracción y equipo especializado; el acceso es complicado por la resistencia a la tracción mediado por las diferentes estructuras anatómicas involucradas y la presión negativa articular. ${ }^{1,2,3}$ A pesar de esto, la cirugía artroscópica de la cadera ha evolucionado en los últimos años, ofreciendo oportunidad de tratamiento a muchas patologías intraarti-

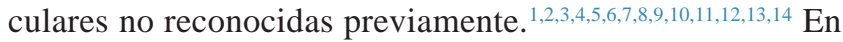
1931, Michael S. Burman fue el primero en realizar una artroscopía de cadera, sin embargo, el procedimiento no ganó popularidad hasta finales de los años 70, y las primeras descripciones del uso de artroscopía de cadera para las lesiones traumáticas se presentaron hasta $1980 .^{1,5}$ Goldman y colaboradores describen la extracción asistida por artroscopía de un proyectil de arma de fuego, alojado en la superficie articular femoral postero-superomedial. ${ }^{9}$ En el mismo año, se reportaron dos informes más de artroscopía en el procedimiento de extirpar fragmentos de metilmetacrilato de un paciente con artroplastía total de cadera. ${ }^{10}$

Hay un aumento de indicaciones que incluyen: diagnóstico de dolor de cadera, lesiones del labrum acetabular, extracción de cuerpos extraños, desbridamiento en artritis séptica, pinzamiento femoroacetabular y algunas lesiones

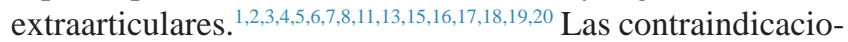

Keywords: Hip arthroscopy, fracture, acetabulum, minimally invasive.

nes son pocas, como el dolor de cadera por causas extraarticulares, osteonecrosis, infecciones de la piel, úlceras, sepsis o condiciones que limiten la tracción articular. ${ }^{3,6,7,8,17,18,19,21}$

En el manejo de fracturas acetabulares simples, 9,10,11,14,15,20,21,22,23,24,25,26,27 el tratamiento de éstas es un área compleja que se está perfeccionando continuamente. Son causadas por mecanismos de alta energía y los daños asociados son frecuentes. ${ }^{10,20,23,24}$ Las fracturas acetabulares y las fracturas-luxaciones de la cadera afectan la estabilidad de esta articulación, la intervención temprana es esencial para mejorar el pronóstico.

En pacientes con trauma múltiple, son comunes las restricciones quirúrgicas por lesiones multisistémicas. Las fracturas complejas pueden requerir procedimientos largos con gran pérdida hemática, así como una exposición extensa, que puede favorecer complicaciones como infección, problemas de cicatrización, debilidad de abductores, parálisis del nervio ciático y osificación heterotópica. ${ }^{1}$

A pesar del avance tecnológico y el incremento del uso de la artroscopía de cadera para diagnosticar y tratar lesiones intraarticulares, las indicaciones en el manejo de fracturas aún no han sido claramente establecidas. ${ }^{10,11,16,20,22}$ Con el uso de la artroscopía se pueden mejorar los factores mencionados con antelación. ${ }^{17,18}$

A medida que la experiencia del cirujano aumenta en artroscopía de cadera, las condiciones tratadas también. ${ }^{17,18,28}$ Ahora es una técnica ortopédica más común y aplicable. Sin
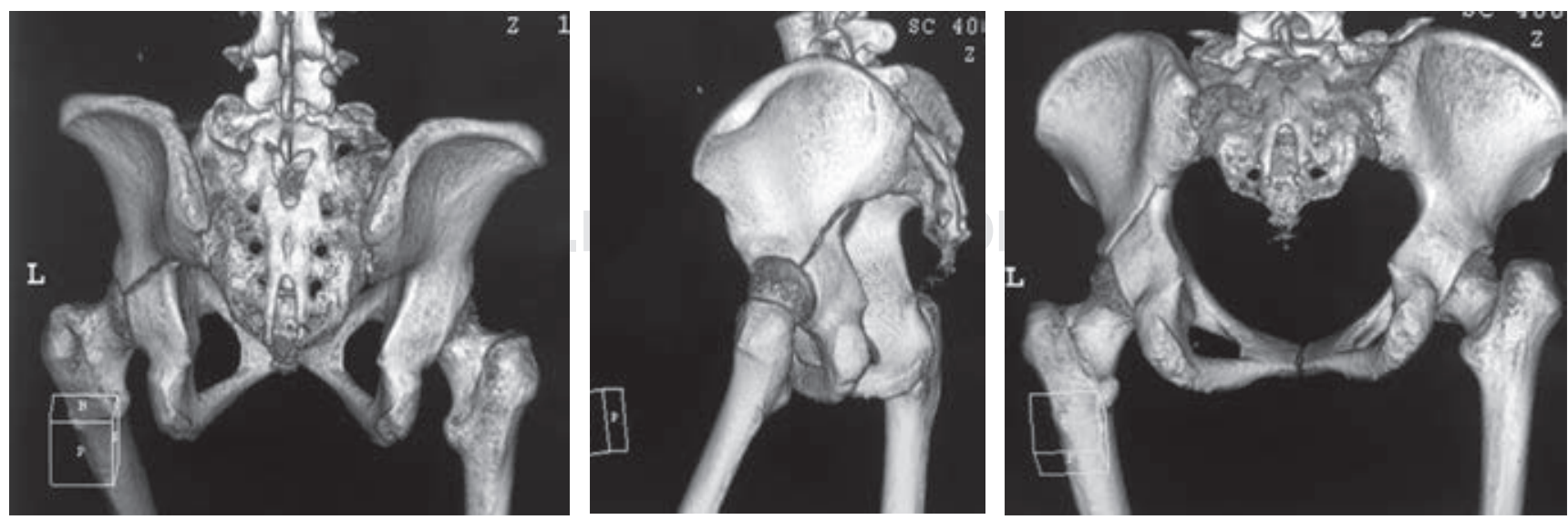

Figura 1: Tomografía axial computada con reconstrucción en 3D, donde se observa trazo de fractura de columna posterior de acetábulo con mínimo desplazamiento. 

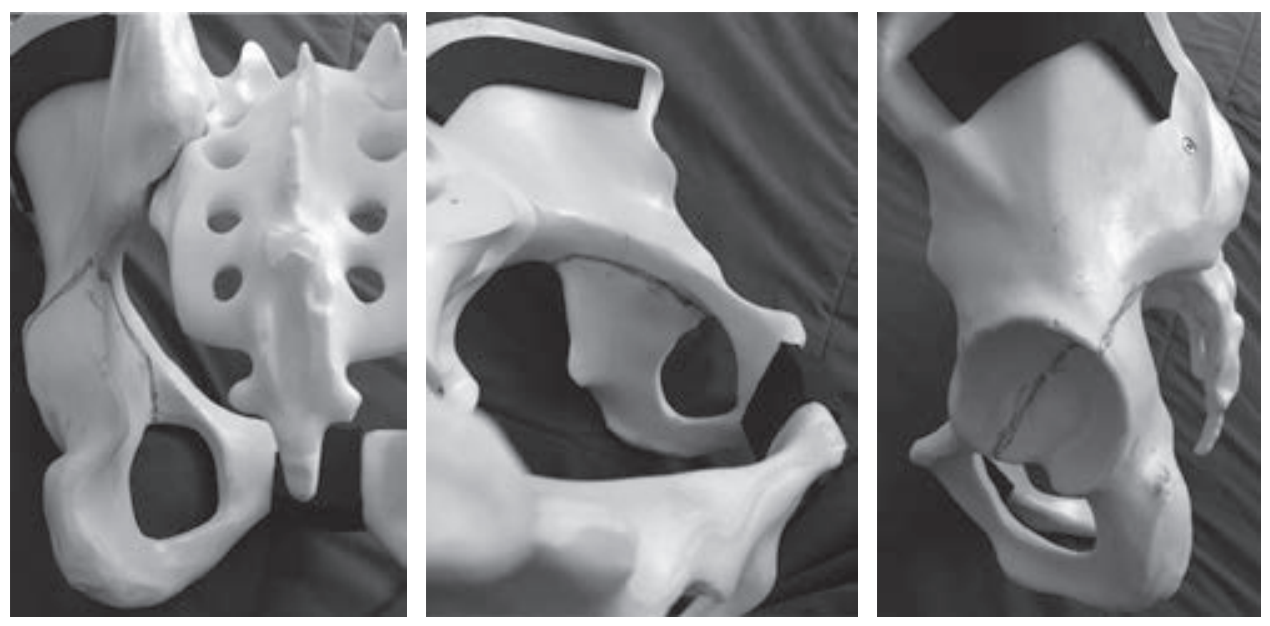

Figura 2:

Planificación preoperatoria en modelo plástico.

embargo, la adquisición de la destreza es un proceso lento y no está exento de complicaciones. ${ }^{1,3,4,5,6,7,17,18,19,21,28}$

El procedimiento artroscópico debe ayudar a proporcionar buena estabilidad, compresión interfragmentaria y menor morbilidad que la cirugía abierta y debe permitir examinar la superficie intraarticular, ayudando en la reducción y favorecer el tratar alguna lesión asociada. ${ }^{20,23,24}$

En 2003, Yamamoto reporta un caso de fijación percutánea asistida por artroscopía en una fractura acetabular en zona de carga. ${ }^{21}$ Yang posteriormente informó sobre dos casos de fracturas de la columna anterior tratadas con reducción indirecta asistida por artroscopía y fijación percutánea, ${ }^{24}$ reportando consolidación y ausencia de cambios artrósicos en un seguimiento a tres años. En 2014, Kim y colaboradores informaron dos casos de reducción y fijación interna artroscópica en dos pacientes jóvenes con fractura de pared posterior y de la columna anterior, respectivamente, con seguimiento postoperatorio a tres meses, asintomáticos y reintegrados a las actividades, con consolidación radiográfica completa a los dos años. ${ }^{23}$ En 2016 Chung refiere que la fijación percutánea mediante fluoroscopio con asistencia artroscópica puede ser más útil en pacientes con traumatismos múltiples, reportaron dos casos de fractura de la pared posterior acetabular, tratados mediante reducción artroscópica y fijación percutánea de tornillos, concluyendo que la técnica es útil para reducir el dolor postoperatorio y lograr el retorno temprano a las actividades para el paciente. ${ }^{22}$

En México, en 2016, Ilizaliturri reporta casos de artroscopía de cadera en el tratamiento de fracturas luxaciones con lesiones de cabeza femoral Pipkin I y II, así como resección de cuerpos extraños en lesiones por arma de fuego. ${ }^{29}$

Los puntos clave para determinar el uso de la artroscopía deben ser que el patrón de fractura sea simple y no desplazado, de manera que afecte de forma importante en la tracción necesaria para la exposición del sitio a trabajar. La visualización en presencia de hemartrosis puede ser difícil y la insuficiencia en la arquitectura ósea para contener el tratamiento artroscópico debe tomarse en consideración. 10,11,15,16,17,18,20,22,23 En este contexto, se reporta el caso con fractura de columna

posterior acetabular tratado vía artroscópica, buscando evaluar la efectividad en el tratamiento.

\section{Caso clínico}

Femenino, de 38 años de edad, con antecedente de accidente automovilístico, con trauma e impacto indirecto
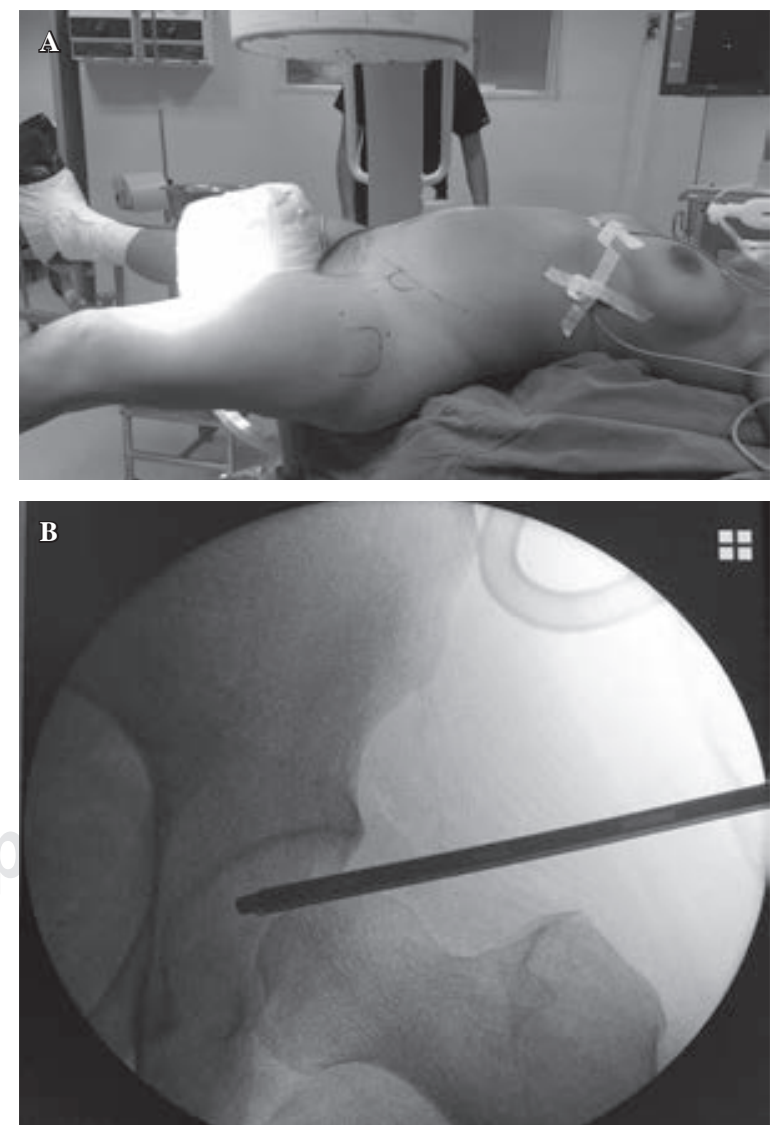

Figura 3: A) Posición del paciente sobre mesa de tracción y colocación de fluoroscopio. B) Visión fluoroscópica de la cadera con subluxación e introducción de lente intraarticular. 


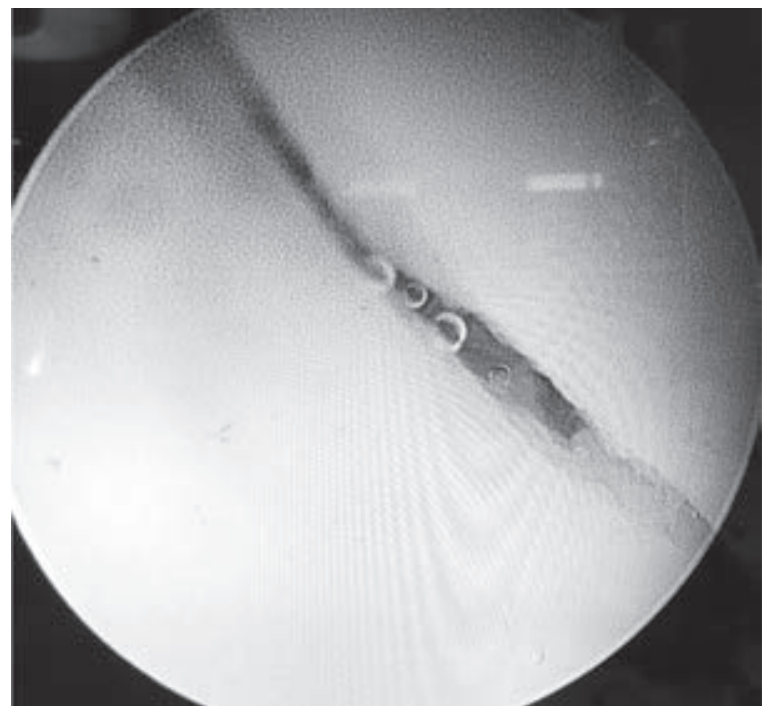

Figura 4: Visión artroscópica de trazo de fractura con separación entre fragmentos de $4 \mathrm{~mm}$.

en rodilla izquierda, causando dolor y limitación funcional de la extremidad pélvica izquierda; fue evaluada en urgencias sin lesiones multisistémicas asociadas. Se realizó toma de las proyecciones radiográficas simples y tomografía axial computada de pelvis, con reconstrucción en 3D (Figura 1). Se observó pérdida de continuidad ósea a nivel de línea iliopectínea, llegando al diagnóstico de fractura simple de acetábulo izquierdo AO 62-A2.2 que involucra la columna posterior. ${ }^{30}$

La paciente fue hospitalizada y programada para su manejo quirúrgico definitivo. Se realizó planificación en modelo plástico (Figura 2) y se decidió realizar reducción cerrada y fijación interna bajo principio biomecánico de compresión interfragmentaria ${ }^{31}$ y asistir reducción bajo visión directa mediante artroscopía de cadera.

Técnica quirúrgica: bajo anestesia general controlada, se posicionó a la paciente en decúbito supino sobre mesa quirúrgica de reducción de fracturas con la extremidad pélvica en tracción, utilizando fluoroscopio para visualizar la articulación coxofemoral (Figura 3).

$\mathrm{Al}$ realizar la tracción de miembro pélvico izquierdo se logró la subluxación con apertura del espacio articular coxofemoral necesario para la introducción del material artroscópico. Por el portal anterolateral se introdujo el lente de 70 grados para visualización intraarticular. Se localizó con visión intraarticular el portal anterior estándar para introducción de material de trabajo. En la exploración articular se observó hemartrosis con sinovitis inflamatoria, se realizó lavado intraarticular y hemostasia, se extrajeron fragmentos libres de cartílago con punta de rasurador. Se localizó lesión traumática de labrum en región posterior acetabular, la cual se estabilizó con radiofrecuencia, se ubicó el trazo de fractura acetabular en columna posterior longitudinal simple con separación de 4 mm (Figura 4). Se realizó abordaje de $4 \mathrm{~cm}$ en primera ventana de Judet y se disecó por planos hasta localizar con guía canulada el punto de entrada para la colocación de tornillo de compresión, asistido por fluoroscopía de $7.0 \mathrm{~mm}$ por $85 \mathrm{~mm}$ y bajo visión directa intraarticular para observar el cierre del trazo de fractura con congruencia articular satisfactoria (Figura 5). Finalmente se procedió a la extracción de instrumental artroscópico y cierre de heridas por planos.

Evolución posquirúrgica: con un movilizador pasivo de cadera se efectuó de manera inmediata en el posquirúrgico la movilización, logrando flexión de 90 grados sin dolor. En el primer día de postquirúrgico se egresó a la paciente con apoyo diferido por ocho semanas. A las 12 semanas, el control radiográfico se observó con adecuada consolidación, la paciente, por completo asintomática, logró realizar la marcha con carga completa y con arcos de movilidad normales en la cadera intervenida. Se aplicó la escala Harris Hip Score, ${ }^{32}$ obteniendo un resultado de 91, considerado como excelente (Figura 6).

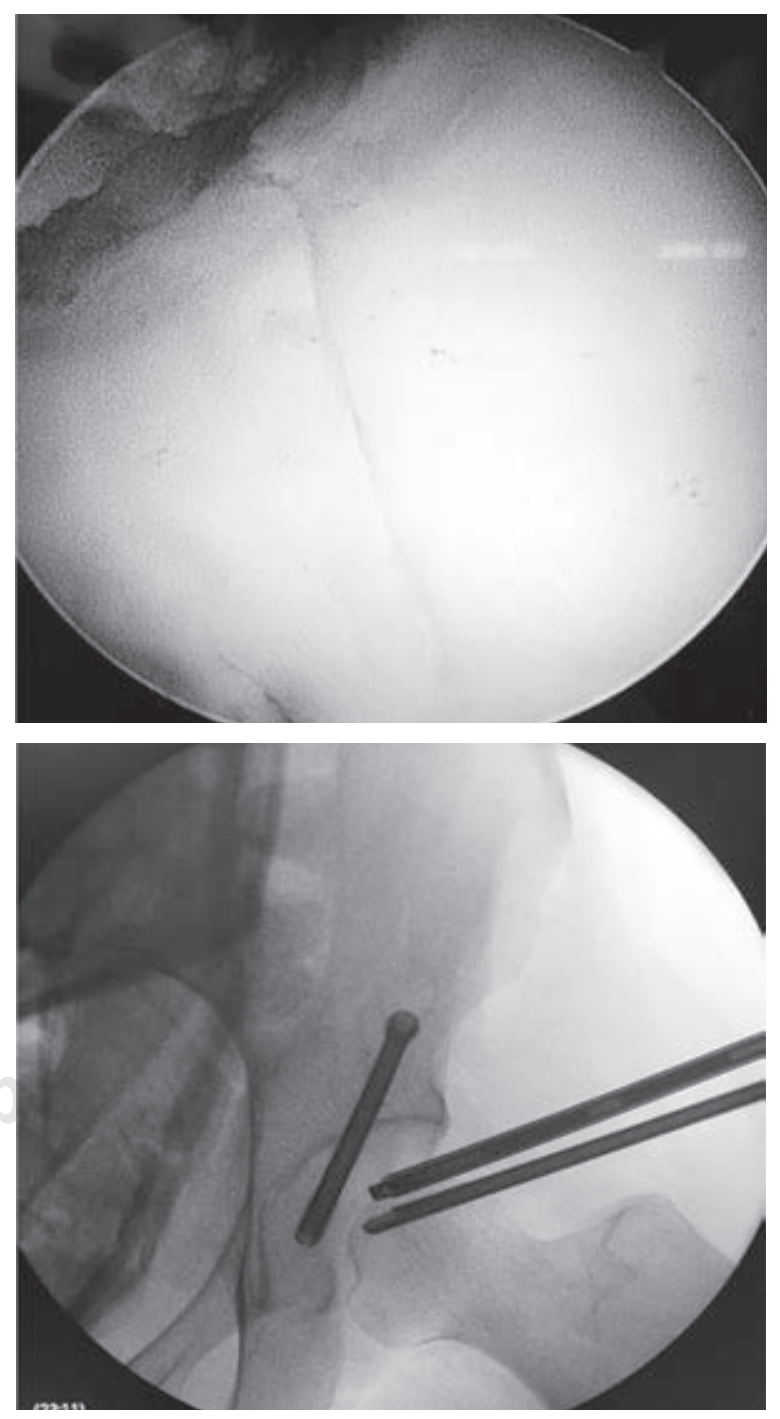

Figura 5: Compresión en el trazo de fractura, donde se observa reducción anatómica al colocar el tornillo de doble compresión. 

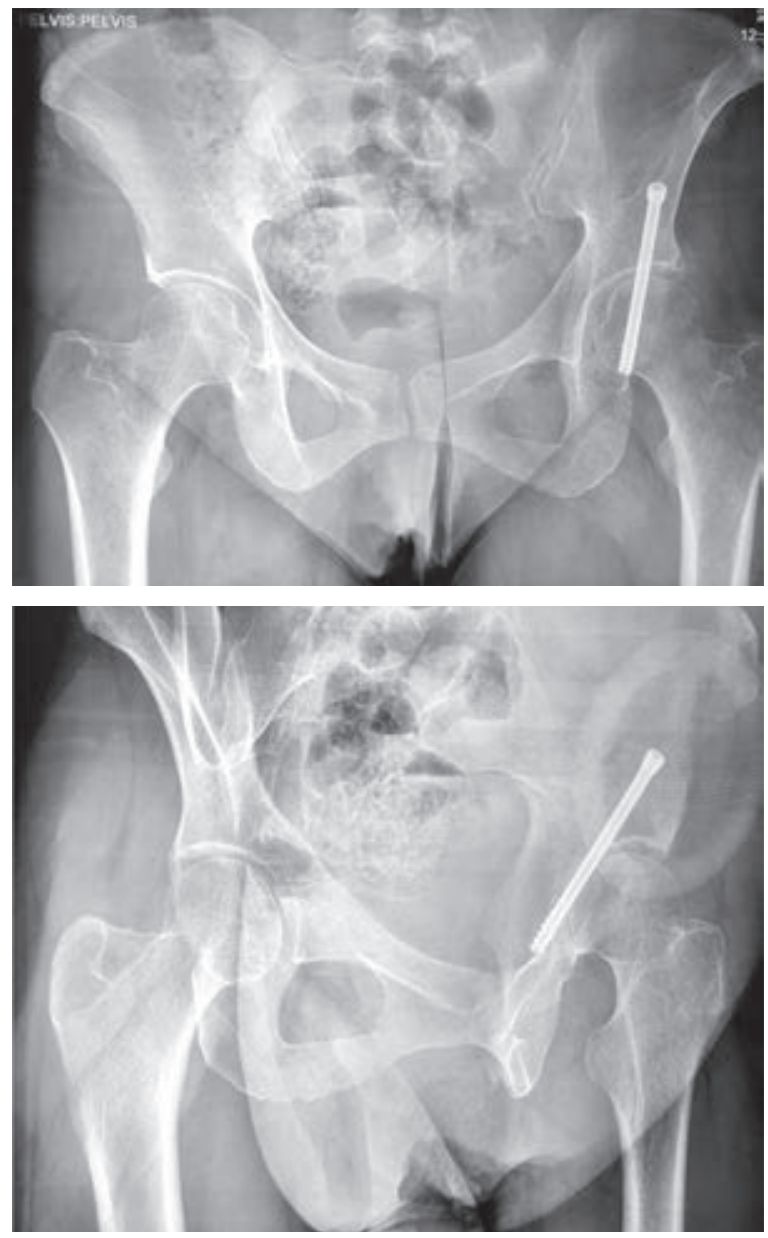

Figura 6: Resultado radiográfico postquirúrgico.

\section{Discusión}

La artroscopía de cadera reduce el daño a músculos, nervios y vasos importantes. Además de tener las ventajas de minimizar el tamaño de las incisiones, poca pérdida sanguínea, rápida movilización articular, pronta recuperación en rehabilitación, el lavado y el desbridamiento de la articulación de la cadera. ${ }^{21,22,23,24}$

Las fracturas de la columna posterior de acetábulo deben tratarse mediante reducción anatómica y fijación, con la finalidad de lograr movilización temprana y minimizar así el riesgo de artrosis postraumática. La fijación interna, temprana y percutánea puede ayudar a evitar complicaciones en pacientes de alto riesgo mediante una cirugía simple y menos invasiva. ${ }^{22}$

Las técnicas artroscópicas para la fijación de fracturas intraarticulares ofrecen la ventaja de una visualización y reducción superiores de la superficie articular permitiendo garantizar una reducción anatómica. Además, permite el tratamiento concomitante de otras lesiones intraarticulares. ${ }^{22}$

Son pocas las indicaciones de la cirugía artroscópica de cadera, en combinación con cirugía percutánea acetabular, pues sólo puede realizarse en aquellos casos de fracturas con mínimo desplazamiento. Los instrumentos artroscópicos para la fijación del acetábulo reducido requieren mejoras adicionales..$^{23}$

El uso de la fijación percutánea del tornillo, es una opción más amigable con los tejidos y debe ser una prioridad. La artroscopía de cadera es de utilidad para asistir durante el proceso de reducción y verificar que el trayecto del tornillo no se encuentre en trayecto intraarticular.

\section{Bibliografía}

1. McCarthy JC, Lee JA. History of hip arthroscopy: challenges and opportunities. Clin Sports Med. 2011; 30(2): 217-24.

2. Tzaveas AP, Villar RN. Arthroscopic repair of acetabular chondral delamination with fibrin adhesive. Hip Int. 2010; 20(1): 115-9.

3. Byrd JW. Hip arthroscopy. J Am Acad Orthop Surg. 2006; 14(7): 433-44.

4. Shetty VD, Villar RN. Hip arthroscopy: current concepts and review of literature. Br J Sports Med. 2007; 41(2): 64-8; discussion 68.

5. Khanduja V, Villar RN. Arthroscopic surgery of the hip: current concepts and recent advances. J Bone Joint Surg Br. 2006; 88(12): 1557-66.

6. McCarthy JC, Lee J. Hip Arthroscopy: Indications, Outcomes, and Complications. J Bone Joint Surg Am. 2005; 87(5): 1137-45.

7. McCarthy JC, Lee J. Hip arthroscopy: indications and technical pearls. Clin Orthop Relat Res. 2005; 441: 180-7.

8. Kelly BT, Williams RJ 3rd, Philippon MJ. Hip arthroscopy: current indications, treatment options, and management issues. Am J Sports Med. 2003; 31(6): 1020-37.

9. Goldman A, Minkoff J, Price A, Krinick R. A posterior arthroscopic approach to bullet extraction from the hip. J Trauma. 1987; 27(11): 1294-300.

10. Newman JT, Saroki AJ, Philippon MJ. Hip arthroscopy for the management of trauma: a literature review. J Hip Preserv Surg. 2015; 2(3): 242-8.

11. Park MS, Yoon SJ, Choi SM. Hip arthroscopic management for femoral head fractures and posterior acetabular wall fractures (Pipkin type IV). Arthrosc Tech. 2013; 2(3): e221-5.

12. Philippon MJ, Schroder E Souza BG, Briggs KK. Hip arthroscopy for femoroacetabular impingement in patients aged 50 years or older. Arthroscopy. 2012; 28(1): 59-65.

13. Bardakos NV, Vasconcelos JC, Villar RN. Early outcome of hip arthroscopy for femoroacetabular impingement: the role of femoral osteoplasty in symptomatic improvement. J Bone Joint Surg Br. 2008; 90(12): 1570-5.

14. Farjo LA, Glick JM, Sampson TG. Hip arthroscopy for acetabular labral tears. Arthroscopy. 1999; 15(2): 132-7.

15. Hwang JT, Lee WY, Kang C, Hwang DS, Kim DY, Zheng L. Usefulness of arthroscopic treatment of painful hip after acetabular fracture or hip dislocation. Clin Orthop Surg. 2015; 7(4): 443-8.

16. Banaszkiewicz PA. Traumatic arthritis of the hip after dislocation and acetabular fractures: treatment by mold arthroplasty: an end-result study using a new method of result evaluation. In: Banaszkiewicz P, Kader D (eds). Classic papers in orthopaedics. London: Springer; 2014. p. 13-7.

17. Harris JD, McCormick FM, Abrams GD, Gupta AK, Ellis TJ, Bach BR Jr, et al. Complications and reoperations during and after hip arthroscopy: a systematic review of 92 studies and more than 6,000 patients. Arthroscopy. 2013; 29(3): 589-95.

18. Oak N, Mendez-Zfass M, Lesniak BP, Larson CM, Kelly BT, Bedi A. Complications in hip arthroscopy. Sports Med Arthrosc Rev. 2013; 21(2): 97-105.

19. Atesok K, Doral MN, Whipple T, Mann G, Mei-Dan O, Atay OA, et al. Arthroscopy-assisted fracture fixation. Knee Surg Sports Traumatol Arthrosc. 2011; 19(2): 320-9.

20. Kim HJ, Kim SS, Jung YH, Lee KH. Effectiveness of hip arthroscopy performed simultaneously before open reduction and internal fixation for acetabular fracture and fracture-dislocation of the hip. Hip Pelvis. 2018; 30(2): 92-100. 
21. Yamamoto Y, Ide T, Ono T, Hamada Y. Usefulness of arthroscopic surgery in hip trauma cases. Arthroscopy. 2003; 19(3): 269-73.

22. Park JY, Chung WC, Kim CK, Huh SH, Kim SJ, Jung BH. Arthroscopic reduction and transportal screw fixation of acetabular posterior wall fracture: technical note. Hip Pelvis. 2016; 28(2): 120-6.

23. Kim H, Baek JH, Park SM, Ha YC. Arthroscopic reduction and internal fixation of acetabular fractures. Knee Surg Sports Traumatol Arthrosc. 2014; 22(4): 867-70.

24. Yang JH, Chouhan DK, Oh KJ. Percutaneous screw fixation of acetabular fractures: applicability of hip arthroscopy. Arthroscopy. 2010; 26(11): 1556-61.

25. Matsuda DK. A rare fracture, an even rarer treatment: the arthroscopic reduction and internal fixation of an isolated femoral head fracture. Arthroscopy. 2009; 25(4): 408-12.

26. Epstein NJ, Safran MR. Stress fracture of the acetabular rim: arthroscopic reduction and internal fixation. A case report. $J$ Bone Joint Surg Am. 2009; 91(6): 1480-6.

27. Singleton SB, Joshi A, Schwartz MA, Collinge CA. Arthroscopic bullet removal from the acetabulum. Arthroscopy. 2005; 21(3): 360-4.
28. Souza BG, Dani WS, Honda EK, Ricioli W Jr, Guimarães RP, Ono NK, et al. Do complications in hip arthroscopy change with experience? Arthroscopy. 2010; 26(8): 1053-7.

29. Ilizaliturri Jr VM, Suárez-Ahedo C. Artroscopía de cadera en patología traumática. Rev Esp Artrosc Cir Articul. 2016; 23(1): 54-62.

30. Polesello GC, Nunes MA, Azuaga TL, de Queiroz MC, Honda EK, Ono NK. Comprehension and reproducibility of the Judet and Letournel classification. Acta Ortop Bras. 2012; 20(2): 70-4.

31. Ramos-Maza E, García-Estrada F, Domínguez-Barrios C, et al. Principios biomecánicos para la osteosíntesis, re-evolución. Acta Ortop Mex. 2016; 30(Suppl: 1): 1-8.

32. Harris WH. Traumatic arthritis of the hip after dislocation and acetabular fractures: treatment by mold arthroplasty. An end-result study using a new method of result evaluation. J Bone Joint Surg Am. 1969; 51(4): 737-55.

Conflicto de intereses: No existe conflicto de intereses para la presentación de este artículo. 